Preprint. Accepted for presentation at the 38th International Symposium on Combustion (delayed to 224-29 January 2021) and accepted July 6, 2020 for publication in the Proceedings of the Combustion Institute, Vol 38.

\title{
Ignition of Stoichiometric Hydrogen-Oxygen by Water Hammer
}

Stephanie A. Coronel*, Jean-Christophe Veilleux, Joseph E. Shepherd California Institute of Technology, Pasadena, CA 91125

\begin{abstract}
The potential of water hammer events for igniting hydrogen-oxygen mixtures was examined in an experimental study. Compression waves simulating water-hammer events were created by projectile impact on a piston in a water-filled pipe terminated by a test section filled with gas. Triangular wave forms with peak pressures up to $50 \mathrm{MPa}$ propagated through the piping system and compressed the gas in the test section. Experiments were carried out with both air and hydrogen-oxygen gas mixtures using highspeed video of the transparent test section, dynamic pressure and spectroscopic measurements to examine the motion of the water-gas interface and determine ignition thresholds. The impulsive acceleration of the water-gas interface and deceleration created by the compression of the gas resulted in Richtmyer-Meshkov and Rayleigh-Taylor instabilities that grew to create large distortions of the initially planar and horizontal water-gas interface. The gas layer was compressed in volume by up to a factor of 50 and the gas pressures increased to as high as $20 \mathrm{MPa}$ within 2 to $4 \mathrm{~ms}$. The distortion
\end{abstract}

\footnotetext{
*Corresponding author:

Email address: scorone@sandia.gov (Stephanie A. Coronel)
} 
of the water surface during compression resulted in a significant increase in interfacial area and ultimately, creation of a two-phase mixture of water and compressed gas. Some ignition events were observed, but the dispersion and mixing of water with the gas almost completely suppressed the pressure rise during the ignition transient. Only by eliminating the instability of the water interface with a solid disk between the water and gas were we able to observe consistent ignition with significant pressure rises associated with the combustion.

Keywords:

Compression Ignition, Richtmyer-Meshkov, Rayleigh-Taylor, Hydrogen

\section{Introduction}

Water hammer [1] is colloquial term for the generation and propagation of pressure transients within piping systems and the associated structural response. In piping systems, pressure transients are usually the result of the sudden opening or closing of valves when the fluid (gas or liquid) within the pipe is flowing rapidly or when a low-pressure piping system is connected by a rapidly opening valve or rupture disk to a high-pressure piping system. In piping systems that contain two-phase regions (liquid-vapor mixtures) or dis-

tinct regions of liquid and vapor, positive pressure transients may cause the compression of the vapor phase, possibly completely collapsing vapor bubbles or a vapor region. When a vapor bubble or region is composed of flammable or explosive vapors, compression may result in temperatures sufficiently high to cause ignition and explosion of the vapor. This is a known hazard [2] in handling liquid propellants and explosives (e.g., hydrazine or nitromethane) 
or industrial scale production of unstable compounds like tetrafluoroethylene [3]. Pressure waves created by the ignition of a vapor bubble or region can propagate into the surrounding liquid explosive and initiate a propagating explosion or decomposition wave with highly destructive consequences [4]. This mechanism has been proposed by Leishear [5] as being relevant to accidents in nuclear power plants.

When a bubble is surrounded by an inert liquid (e.g., a hydrogen-oxygen bubble in water), pressure waves in the liquid can result in individual bubbles exploding $[6,7]$ or in the case of a bubbly liquid, may result in a bubble detonation, a self-sustaining wave of bubble collapse and ignition $[8,9]$. Of particular relevance for the present study are the microdroplets and interfacial area enhancement generated by the interactions of pressure waves with the bubbles. The enhancement of water content due to vaporization can have a significant inhibiting effect on the ignition of the gas within a collapse of a single bubble.

The configuration we examine in this study is closely related to the rapid compression machine (RCM) [10], which uses a piston to rapidly (within 10 - $30 \mathrm{~ms}$ ) compress and hold a reactive mixture at an elevated pressure to enable measurements of autoignition chemistry at pressures and temperatures up to $8 \mathrm{MPa}$ and $1200 \mathrm{~K}$, respectively. The key difference between the present study and previous RCM or shock tube studies of autoignition is that the gas volume is being compressed by a liquid surface that is initially impulsively accelerated by a pressure wave and then continuously decelerated as the pressure in the gas volume increases during compression. This results in a combination of Richtmyer-Meshkov (due to the shock wave) and 
Rayleigh-Taylor (due to the deceleration) instabilities of the liquid-gas interface [11]. Another key difference is that in shock tube or RCM testing, the compressed gas is maintained at nearly constant temperature and pressure over a well defined test time while in the water hammer tests we performed, the compression process is almost immediately followed by an expansion so that the ignition process takes place under highly transient conditions.

The goals of our study were to examine the issues discussed above through an experimental study. We developed a facility to simulate water hammer compression of a gas volume and carried out experiments to characterize the compression of both inert and reactive gas volumes. A set of tests with and without a solid disc (to suppress surface instabilities) demonstrates the dramatic effect of interfacial instabilities on the ignition and combustion process.

\section{Experimental Methodology}

The experiments were performed in a stainless steel U-shaped pipe, shown in Fig. 1 (a), with an inner diameter and thickness of $52 \mathrm{~mm}$ and $4 \mathrm{~mm}$, respectively. A transparent section (test cell) was mounted on a standard stainless steel pipe flange on the right end of the pipe. The flange was 20 mm thick and welded to the pipe end. The test cell was constructed of a hollow, optical-quality polycarbonate cylinder with an inner diameter of 50 $\mathrm{mm}$, outer diameter of $178 \mathrm{~mm}$ and a height of $127 \mathrm{~mm}$. A section of the outside of the test cell was machined flat and vapor polished to improve the quality of visualization of the interior of the cell. The test cell top closure was a $165 \mathrm{~mm}$ diameter, $20 \mathrm{~mm}$ thick stainless steel flange with vacuum 
fittings for a pressure transducer, $P_{\text {cell }}$, and plumbing for filling the cell with the reactive gas mixture. Additional pressure transducers $\left(P_{1}, P_{2}, P_{3}\right.$, and $P_{4}$ ) were placed along the length of the pipe. A vertical gas-gun was used to generate a pressure transient by accelerating downward a projectile which impacted a buffer in contact with the water at the left end of the pipe. A detailed description of the gas-gun and its operation can be found in Inaba and Shepherd [12]. In the present study, the gas-gun accelerates projectiles up to $35 \mathrm{~m} / \mathrm{s}$. The buffer was made of a polycarbonate rod with an outer diameter of $53.3 \mathrm{~mm}$ and height of $152.4 \mathrm{~mm}$. A hole with partial threads was bored through the buffer center axis, making it possible to evacuate the air between the buffer and water. The buffer also had two O-rings to provide gland seals. The projectile was made of a stainless steel rod with an outer diameter of $49.9 \mathrm{~mm}$ and height of $149.2 \mathrm{~mm}$. The buffer and projectile masses, $m_{b}$ and $m_{p}$, respectively, are shown in Fig. 1 (a). A closeup schematic of the test cell is shown in Fig. 1 (b) along with cross-sectional cuts of the test cell configurations: with and without a polypropylene disc separating the gas and water. In the figure, $h_{0}$ is the height of the gas volume. The polypropylene disc had a thickness of $25.4 \mathrm{~mm}$, and two O-rings to provide gland seals. PCB 113B23 high frequency pressure transducers $\left(P_{1}\right.$, $P_{2}, P_{3}, P_{4}$, and $\left.P_{\text {cell }}\right)$ were used in the experiment along with Phantom V710 (2,600 to 88,000 fps) and Phantom V7.3 (12,000 fps) high-speed cameras to image the test cell and projectile-buffer interaction at impact, respectively. An Ocean FX spectrometer was used to measure the optical radiation emitted by the igniting flammable mixture; the spectrometer was only implemented in a few test cases. The cameras and data-acquisition were triggered off of $P_{1}$ 
shortly after the impact of the projectile on the buffer. The pressure signals were digitized with a Yokogawa DL850, 12-bit vertical resolution oscilloscope at $10 \mathrm{MHz}$. A TTL output signal from the oscilloscope was used to trigger the high-speed camera and spectrometer. Details on the experimental procedure can be found in Veilleux et al. [13].

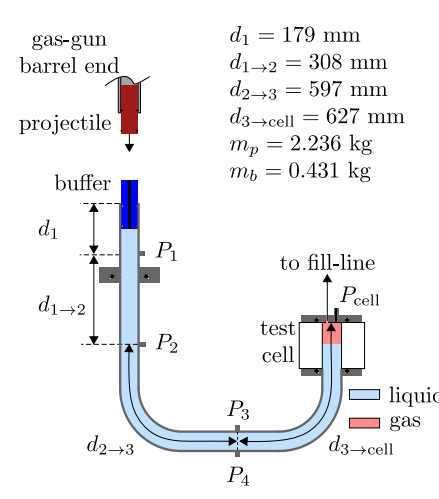

(a) Water-hammer experiment fixture

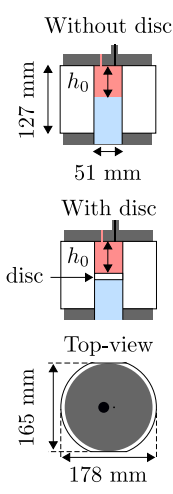

(b) Test cell

Figure 1: Schematics of (a) water hammer experiment fixture and (b) test cell no-disc and disc configurations.

\section{Results}

A total of 32 tests, 18 with and 14 without a plastic disc at the gas-liquid interface were performed with stoichiometric hydrogen-oxygen mixtures. The goals of these tests were to determine the ignition thresholds and characteristics of the resulting combustion events. The targeted nominal height for the reactive gas volume was $100 \pm 3 \mathrm{~mm}$. At this height, 7 tests were carried out with air ( 4 without a disc, 3 with a disc) to serve as a baseline. 


\subsection{Nonreactive results}

Figure 2 (a) shows pressure traces in the liquid column following projectilebuffer impact. Pressure reported in this study refers to the gauge pressure. A primary compression wave is generated, due to the buffer acceleration, that travels from the buffer-liquid interface toward the liquid-gas interface and is followed immediately by expansion waves created as the buffer decelerates [14]. The first pressure waves on transducers $P_{1}, P_{2}, P_{3}$ and $P_{4}$ all consist of a sharp rise (less than $100 \mu \mathrm{s}$ ) followed by a decay to ambient pressure over $1-2 \mathrm{~ms}$. The peak pressure rise in the liquid is $8.9 \mathrm{MPa}$ at $P_{1}, 8.2 \mathrm{MPa}$ at $P_{2}$, and $7.8 \mathrm{MPa}$ at $P_{3}$ and $P_{4}$. The initial magnitude is in agreement with the expected value according to acoustic theory where $P_{\max } \approx \rho c u_{p}=9 \mathrm{MPa}$, $u_{p}$ is the projectile velocity at impact and $c$ is the Korteweg speed [14] of $1380 \mathrm{~m} / \mathrm{s}$, which is slightly less than the liquid sound speed due to the compliance of the piping. At the liquid-gas interface, the compression wave (blue solid line in Fig. 2 (a)) reflects as a tension wave (blue dashed line) due to the larger acoustic impedance of water compared to air. The liquid can not sustain the large negative pressure (tension) and cavitation results. Selected frames from the high-speed video of the test cell are shown in Fig. 2 (b). The first image frame corresponds to the approximate time of the arrival of the primary compression wave at the liquid-gas interface. The passage of a reflected tension wave can be observed by the formation and collapse of bubbles $(t>1 \mathrm{~ms})$. Immediately following the arrival of the compression wave, the liquid column accelerates upwards, shown by the upward movement of the liquid-gas interface, and promptly starts decelerating. At $2.1 \mathrm{~ms}$, the initially planar liquid-gas interface is visibly distorted. This distortion ap- 
pears to originate from the instability of the interface due to a combination of effects: initially Ritchmyer-Meshkov instability due to the incident pressure wave followed by Rayleigh-Taylor instability due to the subsequent deceleration of the interface. The deceleration is caused by the increase in gas pressure due to the compression of the gas volume and decrease in the liquid pressure due to liquid wave dynamics. At $2.7 \mathrm{~ms}$, the interface evolves into a structure consisting of gas bubbles that propagate downward into the liquid and liquid spikes or jets that propagate upward into the gas pocket. At 3.2 ms, the liquid splashes against the top wall of the test cell; this time also corresponds to the first local maximum in $P_{\text {cell }}$. The first local minimum in $P_{\text {cell }}$ is observed at $4.3 \mathrm{~ms}$ corresponding to the expansion of the air volume.

Nonreactive tests showed similar compression pressure-time histories in the test cell with and without the plastic disc, shown in Fig. 3, enabling an evaluation of the ignition thresholds without the potential confounding effects of interfacial instability.

\subsection{Reactive tests with a disc}

Three dilution levels were investigated in reactive gas testing with a disc: $X_{\mathrm{N}_{2}}=\{0,0.25,0.50\}$, where $X_{\mathrm{N}_{2}}$ is the mole fraction of nitrogen. The impact velocity of the projectile on the buffer was varied between 25.0 and $30.0 \mathrm{~m} / \mathrm{s}$, resulting in the production of a primary compression wave with amplitudes of 36.5 to $46.0 \mathrm{MPa}$ in the liquid. In a non-reacting case this results in a peak cell pressure of 9.5 to 27.0 MPa. The results of the experiments performed with the disc are summarized in Fig. 4. The peak pressure on transducers $P_{1}$ and $P_{2}$ as well as the maximum pressure in the cell are shown as a function 

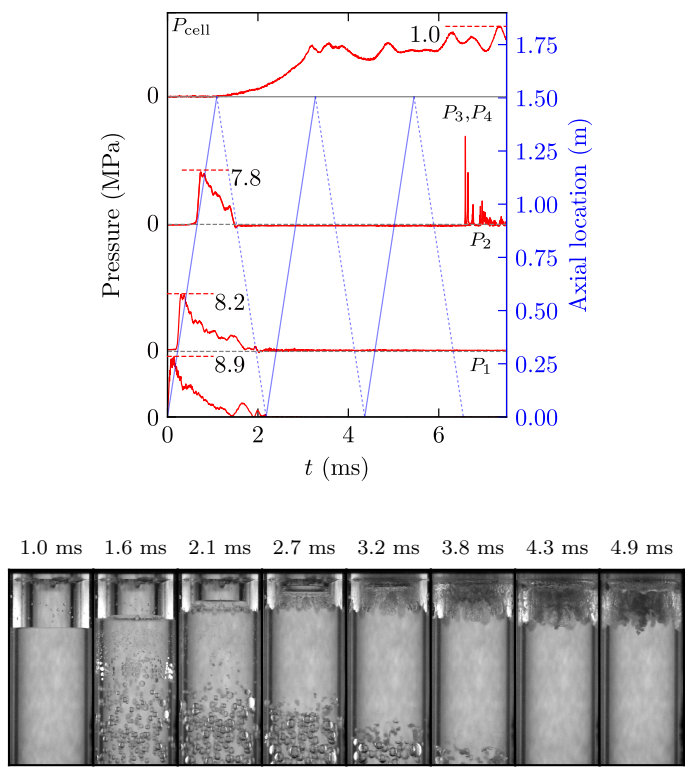

Figure 2: Experimental (a) pressure traces and (b) images of air volume and liquid-gas interface for Test A014; $h_{0}=27 \mathrm{~mm}$ and $u_{p}=6.6 \mathrm{~m} / \mathrm{s}$.

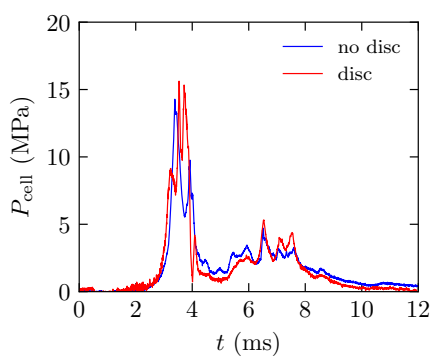

Figure 3: Pressure traces corresponding to $P_{\text {cell }}$ for $h_{0}=100 \pm 1 \mathrm{~mm}$ (Test B044, $u_{p}=$ $28.9 \mathrm{~m} / \mathrm{s}$, disc) and $h_{0}=103 \pm 1 \mathrm{~mm}$ (Test B056, $u_{p}=28.8 \mathrm{~m} / \mathrm{s}$, no disc).

of the impact velocity of the projectile on the buffer. The symbols are coded according to the outcome of the compression event. The peak pressure and 
the luminosity of the combustion event were used to determine if ignition occurred. Ignition was observed in 10 cases, 3 with $X_{\mathrm{N}_{2}}=0.25$ and 7 with $X_{\mathrm{N}_{2}}=0$, and possibly an additional case with $X_{\mathrm{N}_{2}}=0$. The ignition threshold appeared to be at $u_{p}=26 \mathrm{~m} / \mathrm{s}$ corresponding to a peak gas pressure of $10 \mathrm{MPa}$. Ignition was not observed in three tests with $X_{\mathrm{N}_{2}}=0.5$ and one low pressure $\left(P_{\text {cell }}=10 \mathrm{MPa}\right)$ test with $X_{\mathrm{N}_{2}}=0.25$. In the ignition cases (red-filled markers in Fig. 4), the measured peak pressure in the cell is up to 4 times larger than for a non-reacting case and in 4 cases, comparable to or greater than the peak pressure in the initial liquid pressure wave. We suspect that the actual peak pressures in the cell are larger than the peak pressure reported by the transducer: the pressure peaks resulting from the ignition events had an extremely short duration, and were likely not fully resolved in time. Furthermore, the O-ring used to create a seal between the cell and the top flange was not rated for such large pressures; there is evidence which suggests the cell leaks upon ignition. In some cases we observed a high velocity jet of hot steam exhaust leaking through a gap created between the top flange and the test cell body.

An example of an ignition event is Test B060 with a projectile impact velocity $u_{p}=26.8 \mathrm{~m} / \mathrm{s}$ and undiluted stoichiometric mixture $\left(h_{0}=100.1\right.$ $\mathrm{mm}$ ) in the test cell. The pressure recorded in the straight section of the pipe located below the buffer (i.e., $P_{1}$ ) and the pressure in the test cell are shown in Fig. 4 (b) along with an inset of $P_{\text {cell }}$ showing the variation immediately before and after ignition. Note that the pressure data from the test cell transducer is raw (unfiltered) in order to display the sharp peak observed at ignition. The pressure in the cell is $10.0 \mathrm{MPa}$ immediately before ignition, 

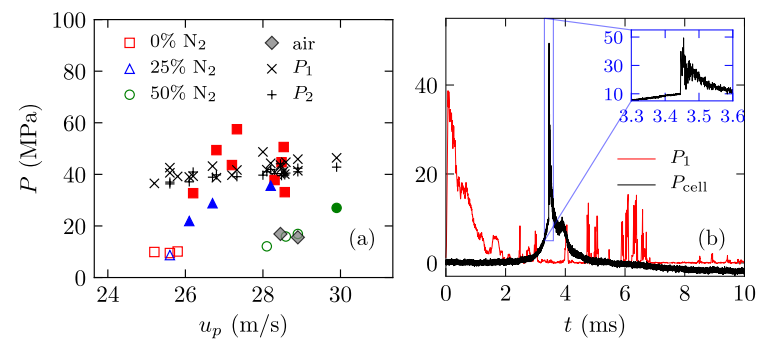

Figure 4: (a) Disc configuration peak pressure and ignition results as a function of projectile velocity for stoichiometric mixtures with three $\mathrm{N}_{2}$ dilution levels; $(\times)$ and $(+)$ are peak $P_{1}$ and $P_{2}$ values, respectively, and the square, triangle, and circle markers are peak $P_{\text {cell }}$ values for reactive mixtures; the closed and open markers are ignition and no-ignition events, respectively; the diamond markers are peak $P_{\text {cell }}$ values for a nonreactive (air) mixture. (b) Pressure in the pipe and test cell during test B060.

and has a peak value of $49.4 \mathrm{MPa}$ immediately after ignition. Note that upon ignition the pressure rapidly increases to its peak value in less than 1 $\mu \mathrm{s}$, similar to the response time of the pressure transducer. This suggests the pressure measurement in the cell could be temporally under-resolved. The rapid increase in pressure in the cell is followed by an equally rapid decay. A few milliseconds after the ignition event the pressure appears to become negative; this is an artifact due to thermal strains produced in the diaphragm of the sensor by the high temperature combustion products. Figure 5 contains a sequence of images showing the test cell and the plastic disc for a short time before and after the ignition event. The times indicated are relative to the trigger event $(t=0)$. The first 6 images $(1.015 \mathrm{~ms}$ to $3.399 \mathrm{~ms})$ correspond to the upward motion of the plastic disc. The ignition event is observed at $3.399 \mathrm{~ms}$. The remainder of the frames correspond to the downward motion 
of the plastic disc (i.e., relaxation of the pressure in the gas volume). The ignition and combustion of the reactive mixture create a substantial amount of chemiluminescence which saturates the sensor of the high-speed camera. Chemiluminescence is visible up to $6.0 \mathrm{~ms}$ after the ignition event.

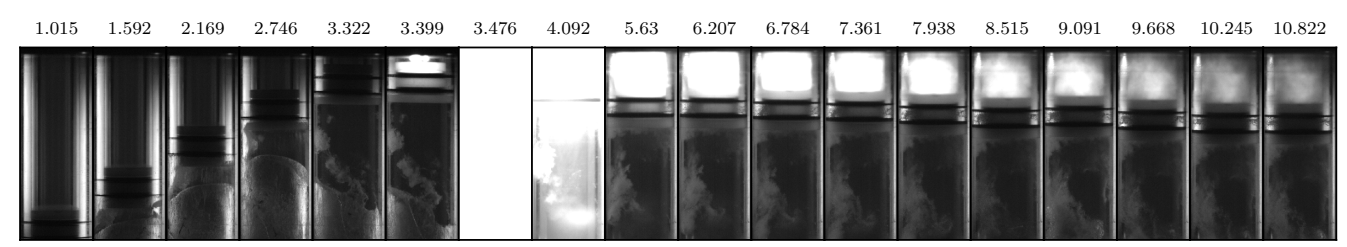

Figure 5: Sequence of images of the test cell for test B060; units are in millisecond.

Figure 6 contains a sequence of images for test B065. The experimental conditions of test B065 are very similar to those of test B060, but the exposure time of the camera was reduced to $0.38 \mu \mathrm{s}$; this is an exposure which is 24 times shorter than the exposure used in test B060. The frame rate of the high-speed camera was also increased. Accordingly, the field of view had to be reduced. The field of view is centered on a region which is very close to the top wall of the cell where ignition is observed. In this configuration it is not possible to distinguish the plastic disc or the test cell prior to the combustion event due to the low light conditions, but it is possible to observe more distinctively the ignition kernel and the flame front upon ignition. Based on the images in Fig. 6 we estimate the flame speed is $1000 \pm 100 \mathrm{~m} / \mathrm{s}$.

The ignition events occurring when the plastic disc separates the reactive mixture from the water all create a substantial amount of chemiluminescence. An Ocean FX spectrometer which can detect light emission between 200 and $1025 \mathrm{~nm}$ was used to measure the emission spectrum of the chemiluminescence. The optical resolution of the instrument is $1.7 \mathrm{~nm}$. A few emission 


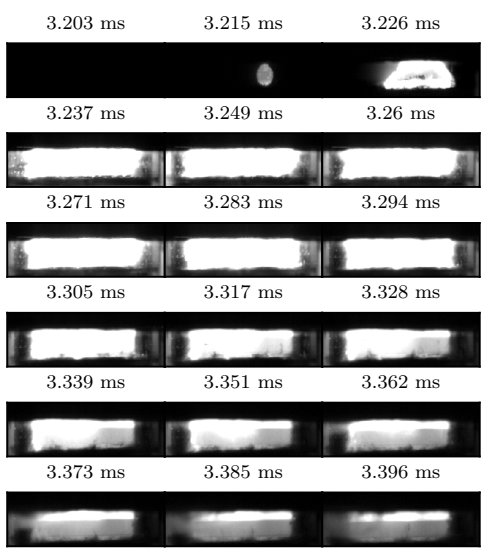

Figure 6: Sequence of images of the top portion of the test cell for test B065.

spectra obtained during test B065 are shown in Fig. 7. The vertical scale corresponds to the relative intensity in arbitrary units. The integration time of each spectrum is $0.1 \mathrm{~ms}$. There is an approximately $0.2 \mathrm{~ms}$ interval between consecutive spectra due to instrument limitations. The emission spectrum of the chemiluminescence consists of strong emission lines superimposed over broadband emission. The light emission between $293 \mathrm{~nm}$ and $322 \mathrm{~nm}$, with two distinctive peaks at $298.0 \mathrm{~nm}$ and $310.6 \mathrm{~nm}$, is likely due to hydroxyl radicals. The strong peak at $593.3 \mathrm{~nm}$ is likely a doublet due to sodium; the resolution of the spectrometer is not sufficient to resolve both peaks. The doublet at $766.3 \mathrm{~nm}$ and $769.5 \mathrm{~nm}$ is likely due to potassium. Sodium and potassium are contaminants which are likely to be found in our test setup.

\subsection{Reactive tests without a disc}

An undiluted stoichiometric mixture was used in 13 tests without a plastic disc separating the reactive gas from the water. One test was carried out 


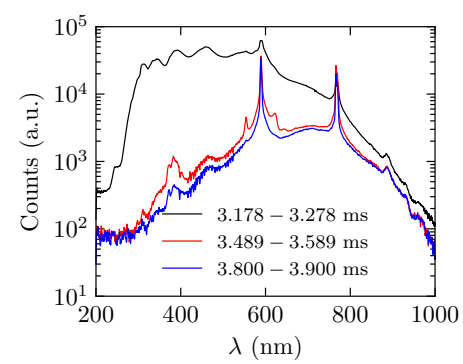

Figure 7: Spectra of the chemiluminescence for test B065.

with $50 \% \mathrm{~N}_{2}$ dilution. All cases had a nominal gas volume height of 100 $\mathrm{mm}$ and projectile impact velocities between 20 and $35 \mathrm{~m} / \mathrm{s}$. A summary of the tests performed without a plastic disc is shown in Fig. 8 which can be directly compared to the disc results shown in Fig. 4 (a). Ignition was determined in these tests by examining the high-speed video for evidence of the characteristic luminosity of combustion and the pressure traces for evidence of the short-duration, high-pressure spikes, both of which were observed in the tests with a disc that had successful ignition. Figure 8 indicates that ignition was observed in five of the tests performed when the projectile velocity was greater that $29 \mathrm{~m} / \mathrm{s}$ and the peak gas pressure was greater than $20 \mathrm{MPa}$. No ignition occurred in 9 tests including three repeat tests at 31 $\mathrm{m} / \mathrm{s}$ impact velocity. Both ignition and no-ignition were observed in tests with projectile velocities between 29 and $31 \mathrm{~m} / \mathrm{s}$. This overlap indicates that there is some variability in the ignition process and ignition probability is more appropriate for describing ignition likelihood than a simple threshold. Based on the testing results with a disc, no effort was attempted to ignite diluted mixtures which would have likely required even higher impact veloc- 
ities than are possible with the present experimental setup. Tests without the disc show two striking differences from the tests with a disc. First, the ignition threshold for undiluted mixtures increased (observation based on a limited number of tests) from an impact velocity of 26 (with disc) to $29 \mathrm{~m} / \mathrm{s}$ (without a disc). Second, the high-pressure ignition peaks observed in the tests with a disc (Fig. 4 (b)) were completely absent in the tests without a disc (Fig. 8). The highest peak pressure observed without a disc was 26.2 $\mathrm{MPa}$ at an impact velocity of $29.1 \mathrm{~m} / \mathrm{s}$. With a disc, the peak pressures ranged from $32-50 \mathrm{MPa}$ at comparable impact velocities.

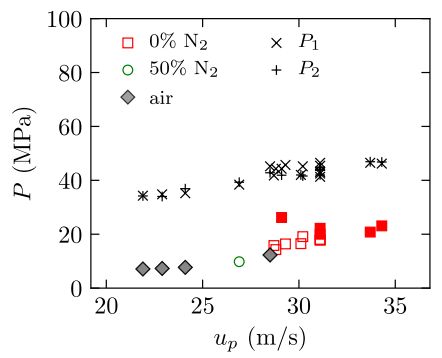

Figure 8: Peak pressure and ignition results as a function of projectile velocity.

The ignition events without a disc resulted in very low levels of luminosity in comparison to those with a disc. Instead of the entire gas region emitting light, emission was very localized and in some regions, entirely absent. An ignition event without the disc is shown in Fig. 9 which contains a sequence of images of the test cell for test B059. The exposure, frame rate, and field of view of the camera is the same as for test B060. The ignition event is observed at $3.188 \mathrm{~ms}$. A small amount of chemiluminescence is visible for 38 $\mu \mathrm{s}$, or one frame. The chemiluminescence visible is confined to a relatively 
small toroidal region adjacent to the test cell wall. We believe this is a direct consequence of the instabilities forming at the interface between the gas and the liquid which result in the formation of a central jet. This jet pushes the reactive mixture to the side of the test cell. With a disc separating the liquid and the reactive mixture the chemiluminescence was observed across the width of the test cell.

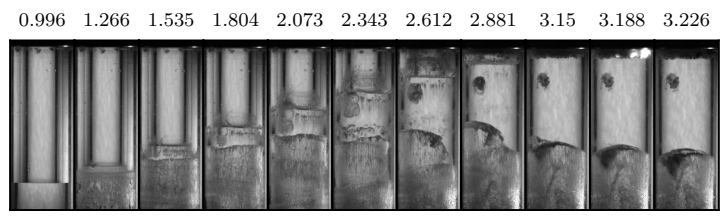

Figure 9: Sequence of images of the test cell for test B059.

The images from Fig. 9 and the small pressure increase also suggest that only a fraction of the reactive mixture initially contained in the cell ignites and burns since a flame was unable to propagate to the left-hand-side of the test cell. Because of the surface instabilities, the reactive mixture appears to be distributed within several pockets which are isolated from one from another. Although ignition occurred within reactive pockets in the liquidgas region, the ignition event was still discernible in the test cell pressure traces. Figure 10 show two different ignition cases corresponding to ignition pressures of 15.4 MPa and 19.6 MPa, respectively. In Fig. 10 (a), the pressure rise due to heat release from the combustion event is approximately $13 \mathrm{MPa}$; however, a relatively smaller pressure rise of approximately $2 \mathrm{MPa}$ is observed in Fig. 10 (b). 

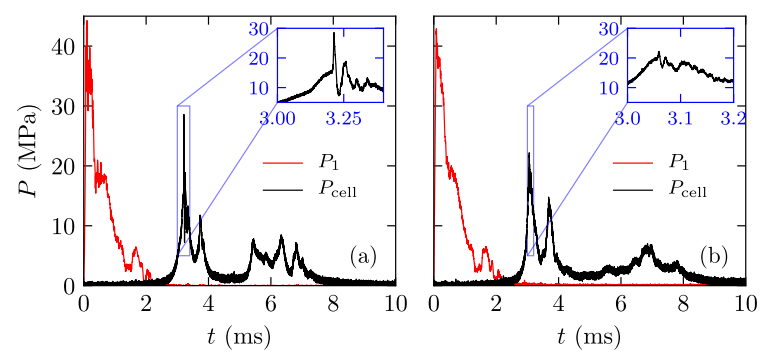

Figure 10: Pressure in the pipe and test cell during (a) test B059 and (b) test B068.

\subsection{Effect of $\mathrm{N}_{2}$ dilution}

Three (B042, B045, B046) of the four cases with 50\% $\mathrm{N}_{2}$ dilution did not obviously ignite (there was no flash of luminosity and the peak pressures were consistent with air compression) in the disc configuration despite using projectile impact velocity velocities up to $29 \mathrm{~m} / \mathrm{s}$ and peak pressures of 17 MPa. Test B041, with $u_{p}=30 \mathrm{~m} / \mathrm{s}$, may have ignited; although there was no flash of luminosity, the peak pressure was $27 \mathrm{MPa}$, much higher than expected based on air compression tests. The combustion event in test B041 may have been masked by the obvious leakage of water past the O-ring seals and into the gas space during the compression phase. The addition of $\mathrm{N}_{2}$ has a negligible effect on the pressure and temperature in the test cell because the specific heat capacity of both diluted and undiluted mixtures is nearly identical. The cell pressure histories for diluted and undiluted cases are very similar for the same projectile velocities. An example is shown in Fig. 11 with nearly identical test parameters with the exception of gas composition. Explosions are observed for 0 and $25 \% \mathrm{~N}_{2}$, but no ignition is observed for $50 \% \mathrm{~N}_{2}$. Of particular note is that the explosion is initiated during the 
compression phase for the most sensitive mixture, after the pressure peak and during the expansion for the less sensitive mixture and not at all for the least sensitive mixture.

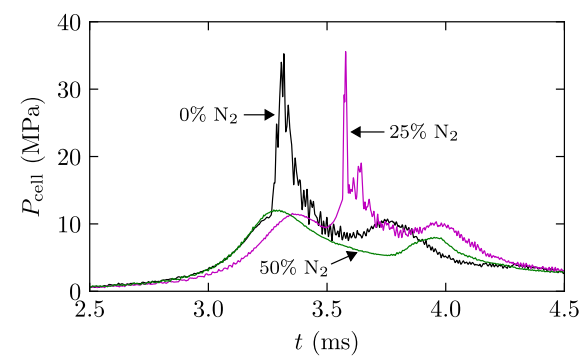

Figure 11: Comparison of three reactive gas disc tests with similar impact velocities and gas volume heights but different amounts of $\mathrm{N}_{2}$ dilution. Test B062, $0 \% \mathrm{~N}_{2}, u_{p}=28.3$ $\mathrm{m} / \mathrm{s}, h_{0}=100.5 \mathrm{~mm}$; test B047, $25 \% \mathrm{~N}_{2}, u_{p}=28.2 \mathrm{~m} / \mathrm{s}, h_{0}=103.2 \mathrm{~mm}$; test B046, $50 \%$ $\mathrm{N}_{2}, u_{p}=28.1 \mathrm{~m} / \mathrm{s}, h_{0}=100.3 \mathrm{~mm}$.

\section{Conclusions}

A key finding in this study is that the interaction of the liquid pressure wave with the liquid-gas interface results in rapid growth of disturbances on the initially planar interface and dispersion of the water into the gas. We attribute the observed interfacial instability to a combination of the impulsive acceleration of the interface (Richtmyer-Meshkov mechanism) and the continuous deceleration of the interface (Rayleigh-Taylor mechanism) occurring during the compression of the gas pocket. By separating the water from the gas with a rigid disc, we were able to demonstrate that the dispersion of the water into the gas significantly influences the ignition threshold and combustion process, suppressing the pressure rise generated by combustion. We also observed that the short duration liquid pressure pulses result in a 
highly transient gas compression event and create the potential for quenching of combustion by rapid expansion that immediately follows compression, even in the cases where the dispersion of water is eliminated. Ignition was possible only when the projectile used to create the water hammer pressure pulse exceeded a threshold velocity of about $26 \mathrm{~m} / \mathrm{s}$ (suppressing mixing with the disc) to $28 \mathrm{~m} / \mathrm{s}$ (with mixing). There are some significant limitations in generalizing the present results to evaluate the potential for reactive gas volume ignition due to water hammer in industrial facilities. We have examined only one configuration of the gas volume and one method of generating the pressure pulse. Our system size is small in comparison to industrial facilities and our method of producing pressure pulses creates a limited range of pressure histories. Industrial facilities such as nuclear power plants have much higher operating pressures and temperatures which have to be taken into account in evaluating the critical conditions for ignition. Despite the significant differences in scale and pressure pulse parameters between our tests and industrial facilities, we anticipate that some of our findings will be universally applicable.

\section{Acknowledgments}

J.-C. Veilleux and S. A. Coronel contributed equally to this work. This work was carried out in the Explosion Dynamics Laboratory of the California Institute of Technology and was partially supported by the US Nuclear Regulatory Commission under Contract NRC-HQ-60-16-T-001. 


\section{References}

[1] E. Wylie, V. Streeter, Fluid Transients in Systems, Prentice Hall, Englewood Cliffs, NJ, 1993.

[2] J. Hershkowitz, B. M. Dobratz, Compendium of Nitromethane Data Relevant to the Tactical Explosive System (TEXS) Program:, Technical Report, Defense Technical Information Center, Fort Belvoir, VA, 1989.

[3] F. Ferrero, R. Meyer, M. Kluge, V. Schröder, T. Spoormaker, Journal of Loss Prevention in the Process Industries 26 (2013) 177-185.

[4] M. W. Ringer, Plant/Oper. Progress 4 (1985) 26-47.

[5] R. Leishear, J. Pressure Vessel Technol. 135 (2013) 054501.

[6] A. E. Beylich, A. Gulhan, in: G. Meier, P. Thompson (Eds.), Adiabatic Waves in Liquid-Vapor Systems, Springer Verlag Berlin Heidelberg, Goettingen, 1990, pp. 3948 .

[7] K. Mitropetros, H. Hieronymus, J. Steinbach, Chemical Engineering Science 61 (2006) 397-416.

[8] T. Hasegawa, T. Fujiwara, Symposium (International) on Combustion 19 (1982) 675683.

[9] A. Pinaev, A. Sychev, Combustion Explosion and Shock Waves 23 (Nov-Dec 1987) $735-742$.

[10] S. Goldsborough, S. Hochgreb, G. VanHove, M. Woolridge, H. Curran, C.-J. Sung, Prog. Energy Combust. Sci. 63 (2017) 1-78.

[11] Y. Zhou, Physics Reports 720-722 (2017) 1-136.

[12] K. Inaba, J. E. Shepherd, in: ASME 2009 Pressure Vessels and Piping Conference, volume 4: Fluid-Structure Interaction, Prague, Czech Republic.

[13] J.-C. Veilleux, S. A. Coronel, J. E. Shepherd, Ignition by Water Hammer, GALCIT Report EDL2019.001, California Institute of Technology, Pasadena, CA 91125, 2019.

[14] J. E. Shepherd, K. Inaba, in: A. Shukla, G. Ravichandran, Y. Rajapakse (Eds.), Dynamic Failure of Materials and Structures, Springer, 2010, pp. 153-190. 